\title{
Exponential stress mitigation in structured granular composites
}

\author{
Andrea Leonard ${ }^{\mathrm{a}, \mathrm{b}}$, Laurent Ponson ${ }^{\mathrm{c}}$, Chiara Daraio ${ }^{\mathrm{a}, \mathrm{b}, *}$ \\ ${ }^{a}$ California Institute of Technology, Department of Aerospace Engineering (GALCIT), 1200 \\ E California Blvd, Pasadena California, CA 91125 USA \\ ${ }^{b}$ ETH-Zurich, Department of Mechanical and Process Engineering \\ (D-MAVT), Tannenstrasse 3 8092 Zurich, Switzerland \\ ${ }^{c}$ Institut Jean le Rond d'Alembert (UMR 7190), CNRS - Université Pierre et Marie Curie, \\ 75005 Paris, France
}

\begin{abstract}
Granular media has been used throughout history as rudimentary yet effective impact mitigation. The unique response of natural granular media is associated with the existence of a network of stress propagation pathways, i.e. a force chain network, which spatially and temporally redirects and moderates the impulse. A variety of structured materials have been proposed to improve the impact mitigating properties compared to natural systems. However, these engineered materials use permanent deformation or viscoelastic properties to dissipate energy, generally limiting their lifetime or effective frequency and temperature range. Here, we take inspiration from natural granular media to engineer a structured composite that exhibits an exponentially fast decay of the leading transmitted pulses. The ordered network geometry allows for an analytical description of the transmitted pulses, which we validate though experiments and numerical simulations. In contrast to other structured materials used for impact mitigation, these networks exhibit reversible deformation, function over all frequencies, and possess a low relative density. Our results open new possibilities for the design and realization of increasingly complex material systems with engineered stress wave transmission pathways.
\end{abstract}

\footnotetext{
* Corresponding author

Email addresses: andreal@caltech.edu (Andrea Leonard), laurent.ponson@upmc.fr (Laurent Ponson), daraio@caltech.edu (Chiara Daraio)
}

Preprint submitted to Extreme Mechanics Letters

December 4, 2014

(C) 2014. This manuscript version is made available under the Elsevier user license http://www.elsevier.com/open-access/userlicense/1.0/ 
Keywords: Network, Non-linear media, Granular materials, Solitary wave, Wave mitigation

\section{Introduction}

Nowadays, material microstructures can be precisely controlled at different scales to achieve new and improved mechanical properties [1, 2, 3]. Here we take inspiration from the advantageous properties of natural granular media, to de-

5 sign and build a structured composite material that employs granular chains to control stress propagation pathways. Granular chains present a unique dynamic response due to both their discrete nature and the nonlinear, Hertzian [4, interaction between particles under compression. For the special case of a uniform particle chain, any axial excitation results in the formation and propagation of nonlinear compact pulses, or solitary waves [5, 6, 7). More generally, granular chains dictate the dynamic behavior of natural, disordered granular packings [8, 9]: instead of the uniform, linear wave propagation observed in a homogeneous solid, a granular medium composed of the same material will transmit excitations through a complex network of force chains [10, 11, 12, 15, 13, 14], i.e. 15 preferred loading paths based on the inter-particle contact network (Fig. 11). This underlying granular chain network controls the response to static forces [10, 11], resistance to intruder impacts [14, 16], and acoustic wave transmission [15, 13. In particular, the amplitude of dynamic excitations are observed to decay exponentially with distance from the impact [17, 15, 14], which makes granular materials highly attractive for wave mitigation applications. However, the primary mechanism for this decay and the local distribution of pulse amplitudes within a granular system are still being investigated. Using an engineered granular network to design structured materials both permits control over the wave propagation pathway, and simplifies the dynamic response allowing for predictive analytical descriptions of the leading pulses. We predict and experimentally observe an exponential decay of the leading pulse amplitudes through our engineered granular network (Fig. 1b and 1 F). This engineered network 
provides insight into the dynamics of natural granular media and allows for the design of new, more efficient stress wave mitigating structures.

The exponential decay rate of the maximum transmitted pulse amplitude is a result of the branched material structure in combination with the nonlinear dynamic response. The branched structure allows for spatial mitigation, and the nonlinear dynamics for temporal mitigation. Under quasi-static loading, the transmitted amplitudes in each segment can be easily calculated from the symmetric amplitude splitting; while the edge (smallest) amplitudes decay exponentially, the central (largest) amplitude decays faster than linear but slower than exponential. Additionally, the transmitted amplitudes for a network geometry composed of a linear media would not differ between quasi-static and dynamic loadings (in the simplest scenario of a non-dispersive media and neglecting edge 4o effects). The amplitude dependent wave speed in our system [5, 6, 7], a property deriving form the nonlinearity, reduces the occurrence of interior wave recombinations, resulting in added temporal wave mitigation of the largest transmitted pulse compared to linear systems. This exponential decay could also be observed in other nonlinear, i.e. non-granular, materials. However, the advantage 45 of using granular chains lies in their inherent ability to break up large amplitude or long duration excitations into a series of smaller pulses [7]. Here, we focus on the relationship between the structured branching geometry and the global dynamic properties in homogeneous granular networks.

The network geometry studied here (Fig. 1p and 1k) was chosen to capture the fundamental physical mechanisms relevant for a general network structure, i.e. multiple wave splittings, bends, and combinations. However, our approach is general and could be used to describe and design an arbitrary force chain structure with variable particles sizes, materials, and network geometries. Previous studies have observed the pulse splitting and combining mechanisms individu5 ally using 2D Y-shaped granular systems [18, 19, 20, 21]. Here, we investigate a 3D branching geometry, which gives rise to multiple occurrences of each mechanism, and investigate the overall stress transmission properties emerging in these types of systems. This study both provides a better comparison with the 
complex force chain structures observed in natural granular media, and allows for improved mitigation performance compared to the $2 \mathrm{D}$ systems recently investigated [22]. Our engineered network incorporates uniform chains of particles only along the predetermined force chain pathways with a supporting matrix filling the remaining 3D volume (Fig $1 \mathrm{p}$ ). This results in a structured material presenting a low effective density compared to densely packed granular media.

\section{2. Materials and Methods}

\subsection{Experiments}

In experiments, the granular networks were constructed from stainless steel sphere chains (type 440C from mcmastercarr.com) held in place by a polymer supporting channel structure, as shown on Fig. 11k. The stainless steel particles were assumed to have an Young's modulus of $E=200 \mathrm{GPa}$, Poisson's ratio of $\nu=0.28$, and a density of $\rho=7800 \mathrm{~kg} / \mathrm{m}^{3}[22$. The particles used in experiments have a manufacturer specified radius of $R=4.7625 \mathrm{~mm}$. The printed VeroClear material used for the supporting channels has a manufacturer specified $\rho=$ $1045 \mathrm{~kg} / \mathrm{m}^{3}$ and Young's modulus $E=2-3 G P a$ (http://objet.com).

For ease in assembly, the supporting channel structure was 3D printed in a modular fashion and then assembled into networks of variable size. To generate a single incident pulse, each granular system was excited by dropping a sphere, identical to those in the network, from a known height. Here, we study the initial pulse propagation along the excited chains within the network, focusing on the leading (largest) pulse amplitudes at each exit branch location. Force measurements were obtained at each of the branch ends with piezoelectric dynamic force sensors (PCB 208C01 and PCB 208C02, with sensitivity $11.2 \mathrm{mV} / \mathrm{N}$ and $112.4 \mathrm{mV} / \mathrm{N}$ ). A conditioner (PCB 481A02) amplified signals when necessary and the data was collected through a data acquisition board (NI BNC-2110 and 85 NI PCI-6123). To relate the measured force at the sensor to the amplitude of the solitary wave traveling through the preceding chain, we used the procedure described by Job et al. [27]. This procedure uses the assumptions that the 
solitary wave kinetic energy is approximately equal to the potential energy and that the potential energy is well described by the most compressed sphere-sphere contact. Since the total solitary wave energy is transferred to potential energy at a sphere-wall interface, the sensor-solitary wave amplitude relation can be reduced to a function of the wall and sphere chain Young's moduli. For the stainless steel impact cap of the sensor and the spheres used in experiments, the relation is $\mathrm{F}_{\mathrm{sw}}=\mathrm{F}_{\text {sensor }} / 1.7$ [27.

\section{Calculations and Theory}

\subsection{Numerical Simulations}

Numerical simulations were performed in 3D based on a conservative discrete particle model, where particles are considered as point masses connected by nonlinear, Hertzian springs [4]. The repulsive force $F_{i j}$ between neighboring spheres $i$ and $j$ evolves as a power law $\delta_{i j}^{3 / 2}$ of their penetration distance. We used a $4^{\text {th }}$ order Runge Kutta scheme to integrate the following system of equations, consisting of: $m_{i} \ddot{\mathbf{u}}_{i}=\sum_{j=1}^{\mathrm{P}} \mathbf{F}_{i j}=\sum_{j=1}^{\mathrm{P}} K_{i j}\left|\boldsymbol{\delta}_{i j}\right|^{3 / 2} \hat{\boldsymbol{\delta}}_{i j}$, where

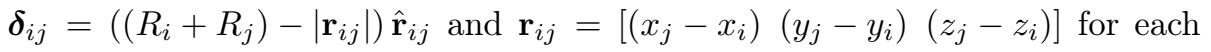
sphere $i$. In these equations, $m$ is the particle mass, $\mathbf{u}_{i}=\left[\begin{array}{lll}u_{i x} & u_{i y} & u_{i z}\end{array}\right]$ represents the particle $x, y$, and $z$ displacement from equilibrium and $P$ is the number of neighboring particles ( $P$ equals $2-4$ spheres plus 3 wall particles). $K_{i j}=\frac{4}{3}\left(\frac{1-\nu_{i}^{2}}{E_{i}}+\frac{1-\nu_{j}^{2}}{E_{j}}\right)^{-1}\left(\frac{R_{i} R_{j}}{R_{i}+R_{j}}\right)^{1 / 2}$ is the contact stiffness between two particles $i$ and $j$ and $\boldsymbol{\delta}_{i j}$ is the penetration distance between two particles. $\boldsymbol{\delta}_{i j}$ takes a zero magnitude when particles $i$ and $j$ are not in contact.The confining walls were modeled as deformable spheres of infinite radius. Three wall particles were placed around the long axis of each bead chain, effectively forming a tube with an equilateral triangular cross-section, limiting particle motion in the out-ofbranch-axis directions. The material properties of the spheres and supporting channel material described in Section 2.1. were used for the numerical simulations. Additionally, a value of $\nu=0.35$, typical for polymers, was assumed for the VeroClear material. 


\subsection{Quasi-Partilce Theory}

The compact nature of the solitary waves allows for each traveling pulse to be equivalently represented by an effective particle, also referred to as the

[22], where detailed derivations were provided, here we present only the required assumptions and main results. The splitting transmission coefficient was derived 
analogously to the 2D configuration [22], but for 3 transmission branches. Similarly, the corner transmission coefficient is identical to the 2D geometry (see 22 ). For 3 identical pulses simultaneously combining into a single transmitted pulse, the transmission coefficient $\mathrm{T}_{\mathrm{M} 3}$ is derived similarly to $\mathrm{T}_{\mathrm{M}}$ in [22], with the assumption that the incident pulses travel at the same branching angle $\alpha$ before and after the merging (a physical constraint imposed by the walls). To derive $\mathrm{T}_{\mathrm{M} 3}$, we follow the same procedure used to derive the merging of 2 chains in the 2D network geometry, and keep only the component of the transmitted quasi-particle velocity along the direction of the transmission branch. The exact expressions for the derived transmission coefficients are:

$$
\begin{array}{cc}
\mathrm{T}_{\mathrm{S}}=\left(\frac{2 \cos \alpha}{1+3 \cos ^{2} \alpha}\right)^{6 / 5}, & \mathrm{~T}_{\mathrm{C}}=(\cos \alpha)^{6 / 5}, \\
\mathrm{~T}_{\mathrm{M} 3}=\left(\frac{6 \cos \alpha}{1+3 \cos ^{2} \alpha}\right)^{6 / 5}, & \text { and } \mathrm{T}_{\mathrm{M} 2}=\left(\frac{8 \sqrt{4-3 \sin ^{2} \alpha}}{3\left(2-\sin ^{2} \alpha\right) \sqrt{4+\tan ^{2} \alpha}}\right)^{6 / 5} .
\end{array}
$$

Numerical simulations were performed on a unit cell network, validating the predicted analytical expressions for the transmission coefficients $\mathrm{T}_{\mathrm{S}}, \mathrm{T}_{\mathrm{C}}, \mathrm{T}_{\mathrm{M} 3}$, and $\mathrm{T}_{\mathrm{M} 2}$ and their variations with the branching angles $\alpha$ (see supplemental material). While the focus of this work is to describe the amplitude of the leading transmitted pulses, the quasi-particle theory could also be used to describe the reflected pulses at splitting junctions [19], and the energy absorbed by the sphere-wall contact at corner junctions [22].

\section{Results and Discussion}

The spatial distribution of pulses propagating through the network is described by $\mathrm{F}_{\mathrm{t}}(\mathrm{r}, \mathrm{N})$, that represents the transmitted pulse amplitude in a network of branching level $\mathrm{N}$ at radial distance $r$, where $r$ varies from 0 to 1 (Fig. 2p). To quantify the performance of such an engineered wave mitigating system, we introduce $\mathrm{F}_{\mathrm{m}}$ that gives the largest amplitude pulse reaching the exit chains opposite the impact, and investigate its variation with distance from the impact, or equivalently, with the network branching level $N$. The largest pulse is always located in the central exit branch, so $F_{m}=F_{t}(r=0, N)$. Following the same 
procedure as ref. [22], we track an incident pulse through the branched structure and obtain the expression of the wave amplitude in each chain segment. The leading pulse amplitudes in each branch of the granular networks with level $N=1-3$ are depicted in Fig 2 and $2 \mathrm{~d}$ for a unitary incident pulse amplitude. The general expressions for the wave amplitude in each branch at location $r$ in a network of size $N$ are calculated exactly in terms of the transmission coefficients, from which the following simplified, approximate expressions for $\mathrm{F}_{\mathrm{m}}$ and $\mathrm{F}_{\mathrm{t}}$ are derived:

$$
\mathrm{F}_{\mathrm{m}}=\mathrm{F}_{\mathrm{i}} e^{\frac{-\mathrm{N}}{\mathrm{N}_{0}}} \text { and } \mathrm{F}_{\mathrm{t}}=\mathrm{F}_{\mathrm{m}} e^{\frac{-\mathrm{Nr}}{R_{0}}}
$$

$\mathrm{F}_{\mathrm{i}}$ denotes the incident pulse amplitude. A detailed derivation of Equation 2 is given in the supplemental material. These expressions capture well the decay of the maximum wave amplitude and the distribution of leading pulse amplitudes predicted by the quasi-particle theory (Fig. 3a-c). The decay constants, $\mathrm{N}_{0}$ and $R_{0}$, given in supplemental material, can be derived from the transmission coefficients, and so depend on the branching angle $\alpha$ only.

Experiments were performed for branching levels, $\mathrm{N}=1,2$ and 3 , using a branching angle, $\alpha=40^{\circ}$, and granular chains made of 5 particles. The dynamic force measurements from experiments are in excellent agreement with the numerical simulations in terms of the number of pulses and the relative amplitude of pulses reaching each branch end (see supplemental material). This indicates that slight misalignments of the granular network are not significant enough to break the symmetry of the system, which would have prevented pulse recombination. While network geometries could be designed to prevent pulse recombination, thus increasing the mitigation efficiency, the focus of this work is to validate the response of a general network.

Fig. $3 \mathrm{~d}$ compares the experimental values of $\mathrm{F}_{\mathrm{m}}$ with numerical results and theoretical predictions. The experimental distribution of transmitted force amplitudes $\mathrm{F}_{\mathrm{t}}$ is normalized by the corresponding $\mathrm{F}_{\mathrm{m}}$ values for each branch level $\mathrm{N}$, and compared with numerical simulations and theoretical predictions in Fig. 3 df. The presence of dissipation in the experimental system (see supplemental 
material) further improves mitigation compared to the already rapid decay of leading pulses resulting from the branched structure. However, since this effect was not taken into account in the model, the experimental wave amplitudes (Fig. 22) are systematically lower than the predicted ones (Fig. 35). Overall, the agreement between the experiments, numerical simulations, and theoretical predictions is quite good, confirming both the exponential decay of the central $\mathrm{F}_{\mathrm{m}}$ amplitude with $\mathrm{N}$ and the exponential decay profile of wave amplitude along $r$ at a given branching level N. For the $\alpha=40^{\circ}$ network, the theoretical values $\mathrm{N}_{0} \simeq 1.8$ and $R_{0} \simeq 1.9$, suggest that the $\mathrm{N}=2$ granular network already results in strong wave amplitude mitigation. The numerical simulations and experiments agree reasonably well with the predicted values (Fig. $3 \mathrm{~d}$-f and supplemental material). However, similar to the observations made in 2D networks [22], the comparison with theory improves as the branch lengths increase, which allows primary and trailing pulses sufficient time to fully separate, as assumed in the model.

We compare now the performance of the engineered granular network with other materials in two ways: (i) efficiency of energy spreading compared to a homogeneous continuum (Fig. 4a), and (ii) attenuation length, $\xi$, compared to viscoelastic materials which also display exponential damping (Fig. $4 \mathrm{~b}$ ) (see the supplemental material for a detailed analysis). In a homogeneous, linear elastic solid, the acoustic energy decreases as a power of the distance from the excitation, as the wave front spreads radially. Fig. 4 s shows that the nonlinear dynamic behavior of our structured granular network spreads the acoustic wave front energy in a more efficient way, especially for larger branching levels. However, unlike uniform viscoelastic solids, the properties of our network are anisotropic. Thus the gain in decay rate of our system is at the expense of a directional dependent response. In the Ashby diagram of Fig. $4 \mathrm{~b}$, the materials located in the top right hand corner are the most efficient acoustic wave mitigators: they combine a small value of $\xi$ with a low effective density. The tested network surpasses designed high damping materials like InSn based composites [24], polyurethane [25] and complex composite polymers [26], that, 
in addition, only function over a small range of frequencies and temperatures.

235 While the attenuation length of disordered and ordered granular packings are of the same order (a few grain diameters [17, 15]), indicating a similar decay mechanism, the effective density is considerably smaller in the engineered network.

In conclusion, we took inspiration from the advantageous properties of natural granular media to engineer a novel structured composite. This system displays the same exponential force reduction observed in disordered granular media, but with added benefits of a low relative density and a predictable response. This response is a result of the structured branching geometry combined with the nonlinear dynamics, and does not require viscoelasticity or permanent 245 deformation to achieve the force reduction. This networked material concept and its analysis is general and could be used to describe more complex systems with customized stress wave pathways, including e.g. splitting and combining processes at junctions made of chains with different material properties and unequal branching angles (see ref. [19]). Additionally, the network structure could be combined with existing 1D engineered granular mitigation techniques [7. The good agreement of theoretical and numerical predictions with experiments for our network topology suggests that more complex networks could be designed using a similar approach, and then fabricated for achieving improved and optimized acoustic properties. Our results both provide insight into the dynamics of natural granular media and open new possibilities for the design and realization of increasingly complex material systems.

\section{Acknowledgments}

This work was supported in part by the Department of Energy Office of Science Graduate Fellowship Program (DOE SCGF), made possible in part by the American Recovery and Reinvestment Act of 2009, administrated by ORISEORAU under contract DE-AC05-06OR23100. We also acknowledge support from the MURI Grant No. US ARO W911NF-09-1-0436. L. Ponson acknowl- 
edges the support of the European Union through a Marie Curie Grant (CIG294025 ToughBridge).

[10] Liu, C.-h. et al. Force fluctuations in bead packs. Science 269, (1995) 513.

[11] Majmudar, T. S. \& Behringer, R. P. Contact force measurements and stress-induced anisotropy in granular materials. Nature 435, (2005) 10791082.

285 [12] Bardenhagen, S. G. \& Brackbill, J. U. Dynamic stress bridging in granular material. J. Appl. Phys. 83, (1998) 5732-5740. 
[13] Bassett, D. S., Owens, E. T., Daniels, K. E. \& Porter, M. A. Influence of network topology on sound propagation in granular materials. Phy. Rev. E 86, (2012) 041306.

[14] Clark, A. H., Kondic, L. \& Behringer, R. P. Particle scale dynamics in granular impact. Phys. Rev. Lett. 109, (2012) 238302.

[15] Owens, E. T. \& Daniels, K. E. Sound propagation and force chains in granular materials Europhys. Lett. 94, (2011) 54005.

[16] Katsuragi, H. \& Durian, D. J. Unified force law for granular impact cratering. Nat. Phys. 3, (2007) 420-423.

[17] Liu, C.-h. Spatial patterns of sound propagation in sand Phys. Rev. B 50, (1994) 782.

[18] Shukla, A., Zhu, C. Y. \& Sadd, M. Angular dependence of dynamic load transfer due to explosive loading in granular aggregate chains. J. Strain Anal. Eng. Des. 23, (1988) 121-127.

[19] Ngo, D., Fraternali, F. \& Daraio, C. Highly nonlinear solitary wave propagation in y-shaped granular crystals with variable branch angles. Phys. Rev. E 85, (2012) 036602.

[20] Daraio, C., Ngo, D., Nesterenko, V. F. \& Fraternali, F. Highly nonlinear pulse splitting and recombination in a two-dimensional granular network. Phys. Rev. E 82, (2010) 036603.

[21] Qiong, C., Xian-Qing, Y., Xin-Yin, Z., Zhen-Hui, W. \& Yue-Min, Z. Binary collision approximation for solitary waves in a Y-shaped granular chain. Chin. Phys. B 22, (2013) 014501.

[22] Leonard, A., Ponson, L. \& Daraio, C. Wave mitigation in ordered networks of granular chains. J. Mech. Phys. Solids (in press). 
[23] Job, S., Melo, F., Sokolow, A. \& Sen, S. Solitary wave trains in granular chains: experiments, theory and simulations. Granular Matter 10, (2007) 13-20.

[24] Ludwigson, M. N., Lakes, R. S. \& Swan, C. C. Damping and stiffness of particulate SiC-InSn composite. J. Comp. mat. 36, (2002) 2245.

[25] Mott, P. H., Roland, C. M., \& Corsaro, R. D. Acoustic and dynamic mechanical properties of a polyurethane rubber. J. Acoust. Soc. Am. 111, (2002) 1782-1790.

[26] Li M., Cheng Y., Xu, Y. \& Qin Y. New damping materials by fabrication of ACM/PVC alloy into hollow fibers. J. Appl. Pol. Sci. 129, (2012) 13341339 (2012).

[27] Job, S., Melo, F., Sokolow, A. \& Sen, S. How hertzian solitary waves interact with boundaries in a 1d granular medium. Phys. Rev. Lett. 94, (2005) 178002 . 
(a)

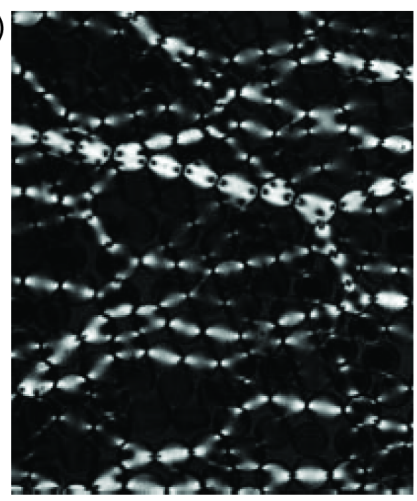

(b)

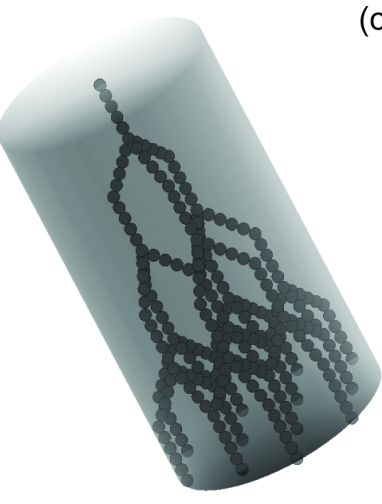

(c)

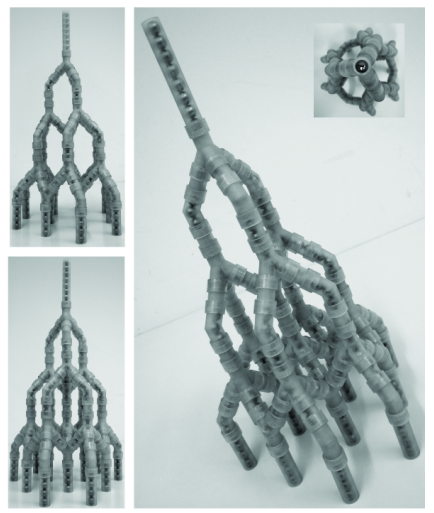

Figure 1: Granular networks. (a) Example of force chain network in disordered granular packings (image reproduced and adapted with permission from [11). The light regions within the packing of photoeslastic cylinders indicate higher stresses. (b) Conceptual schematic showing the engineered granular network, grey spheres, fully embedded in a supporting matrix, shaded cylinder. (c) Photographs of the actual granular network used in experiments. To minimize 3D printed material usage, the supporting matrix was reduced to only the necessary confining channel structure. 
(a)

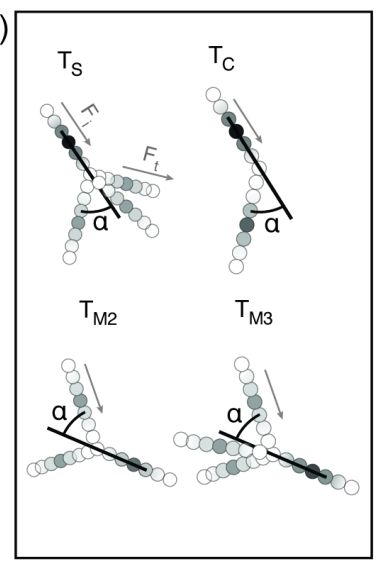

(b)

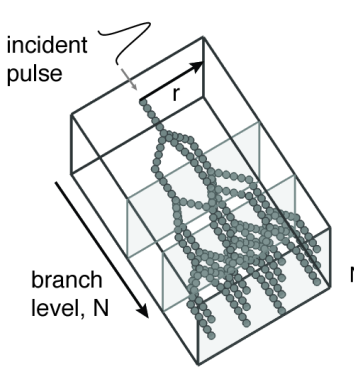

(c)

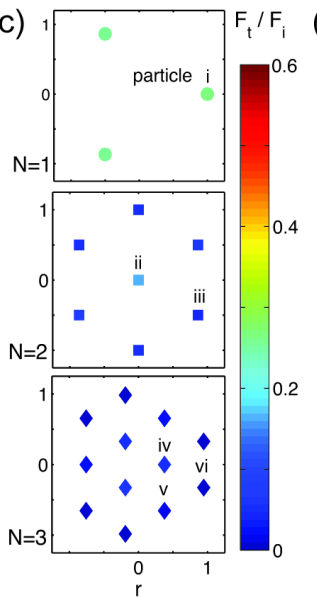

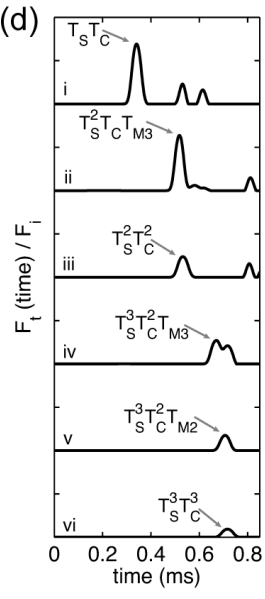

Figure 2: Pulse propagation through the granular network. (a) Schematic diagrams depicting the transmission coefficients, $\mathrm{T}=\mathrm{F}_{\mathrm{t}} / \mathrm{F}_{\mathrm{i}}$, within the network structure. (b) Schematic diagram of granular network geometry describing the branch level, $\mathrm{N}$, and the radial distance from the line of impact, r. (c) Exit branch locations and corresponding average transmitted amplitudes from repeated experiments of $\mathrm{N}=1,2$, and 3. (d) Numerical simulation results, for branch levels $\mathrm{N}=1,2$, and 3 , showing the spatio-temporal structure of the transmitted wave at the exit branch location shown in (c). The amplitude of each leading pulse, with respect to the incident pulse amplitude, is given in terms of the derived transmission coefficients (profiles are offset by $0.5 \mathrm{~N} / \mathrm{N}$ for visual clarity, and y-ticks are $0.25 \mathrm{~N} / \mathrm{N}$ ). 

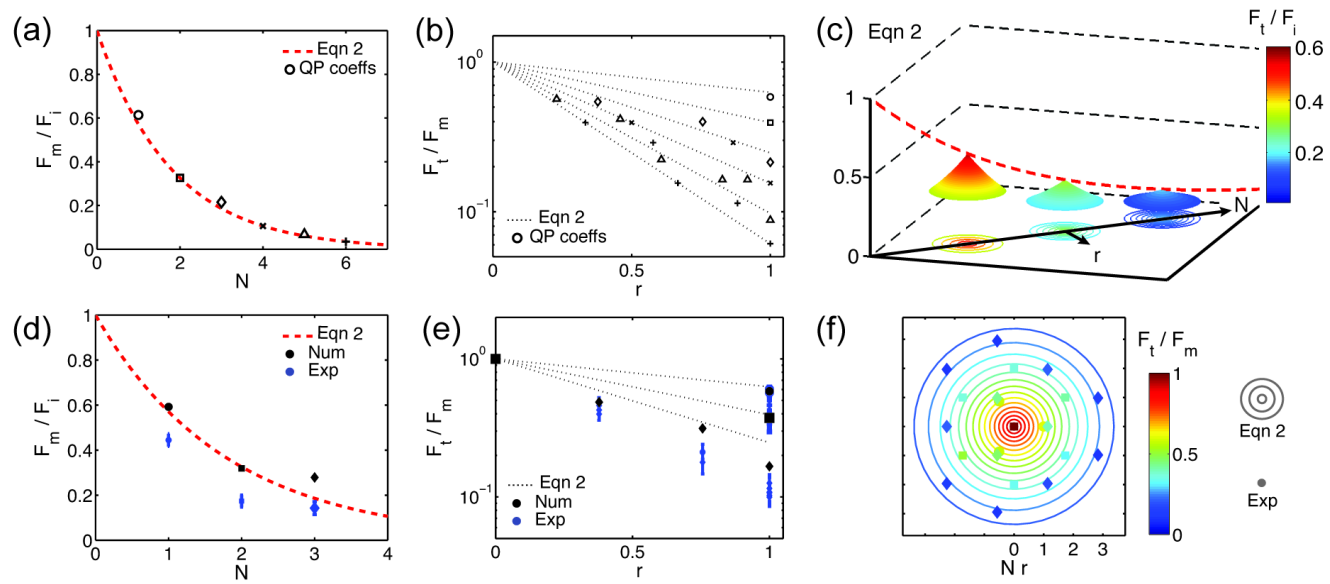

Figure 3: Comparison of QP Theory, Eqn. 22, Experiments and Numerical Simulations. (a-

c) Theoretical predictions of the pulse amplitude: The exact calculation of the normalized amplitudes based on quasi-particle theory is represented with unfilled markers, and is well captured by Eqn. 20 shown in dashed red lines (a) and dotted black lines (b). (c) Macroscopic acoustic performance of the network as predicted by quasi-particle coefficients. The color bar indicates the transmitted primary pulse amplitudes normalized by the impact force, $\mathrm{F}_{\mathrm{t}} / \mathrm{F}_{\mathrm{i}}$. (df) Comparison of experiments (blue markers with error bars), numerical simulations (black filled markers), and theoretical predictions (Eqn. 20), for: (d) the normalized central pulse amplitude $\mathrm{F}_{\mathrm{m}} / \mathrm{F}_{\mathrm{i}}$ as a function of the branch level $\mathrm{N}$, and (e-f) the spatial distribution of leading pulse amplitudes. For (a-f), the marker shapes indicate the branch level N: $\circ(1), \square$ $(2), \diamond(3), x(4), \triangle(5)$ and $+(6)$. 

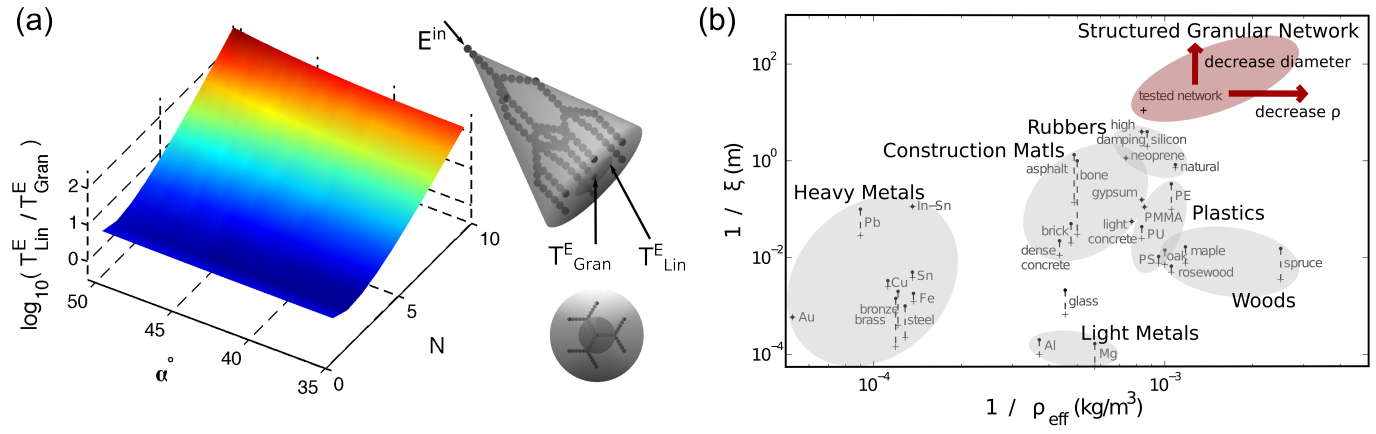

Figure 4: Comparison of stress attenuation performance. (a) Comparison of energy spreading between our engineered network and an equivalently sized cone of homogeneous, linear elastic material. $\mathrm{T}_{\mathrm{Gran}}^{\mathrm{E}}$ is the transmitted energy in the primary pulse of the central exit chain of a granular network of size N. $\mathrm{T}_{\mathrm{Lin}}^{\mathrm{E}}$ is the transmitted acoustic energy in the neighborhood of the central branch after radial spreading. Refer to the supplemental material for a detailed analysis. (b) Inverse of the attenuation length, $1 / \xi$, as a function of the inverse effective density, $1 / \rho$, for a broad range of materials and systems. The $\bullet$ and + connect by a dashed line represent the lower and upper values of $\xi$ (calculated for $f=1 \mathrm{kHz}$ ) from the literature (see supplemental material). The point denoted "tested network" indicates the structured granular network geometry used in experiments, where the effective density was conservatively calculated assuming a solid cylindrical matrix as shown in Fig. 11?. 